\section{CARACTERÍSTICAS DE LA ENFERMEDAD TUBERCULOSA EN ESTUDIANTES DE CIENCIAS DE LA SALUD EN PERU}

\section{CHARACTERISTICS OF TUBERCULOSIS DISEASE IN HEALTH SCIENCE STUDENTS IN PERU}

\author{
Marcelo Chávez-Ramos ${ }^{1, a}$, César V. Munayco ${ }^{1,2, b}$, \\ Gabriela Soto-Cabezas ${ }^{2, b}$
}

Sr. Editor. La tuberculosis (TB) es una enfermedad que afecta tanto a trabajadores de salud como a la población en general (1). No obstante, el riesgo de infectarse y desarrollar la enfermedad tuberculosa en trabajadores de la salud es mucho mayor (2). En un estudio realizado en Perú en un hospital nacional indicó que el personal de salud (población de estudio comprendido por internos y residentes) presentó una tasa anual de conversión de la prueba cutánea de tuberculosis del $17 \%{ }^{(3)}$. En cambio, la tasa de conversión de personas en personas que viven en un entorno urbano pobre y superpoblado fue del $3 \%{ }^{(4)}$. Por ello, se reconoce que el trabajador de salud presenta mayor riesgo de infectarse y presentar la enfermedad tuberculosa, siendo el estudiante de salud uno de los afectados.

El objetivo de esta comunicación es mostrar resultados preliminares sobre las características de la enfermedad tuberculosa en estudiantes de salud. Para ello, se realizó un estudio transversal, usando la base de datos nacional de la Vigilancia epidemiológica de tuberculosis del Centro Nacional de Epidemiología, Prevención y Control de Enfermedades entre los años 2013 y 2016. En este sistema de vigilancia epidemiológica se notifica sistemáticamente los casos nuevos de enfermedad tuberculosa. Cada caso nuevo se registra en una ficha epidemiológica de casos y eventos de tuberculosis, la cual es registrada en un aplicativo electrónico por personal del establecimiento de salud donde se realizó el diagnóstico.

En el estudio se muestra que, de un total de 108 casos nuevos de tuberculosis en estudiantes de ciencias de la salud, el año 2015 presentó el mayor número de casos nuevos. Asimismo, las mujeres representaban $68,5 \%$ de la población de estudiantes de ciencias de la salud y la edad promedio de la población era de 23 años. Además, la carrera universitaria más afectada era la de medicina (Tabla 1).

En cuanto a la localización de la tuberculosis se encontró 81 casos de tuberculosis pulmonar y 27 de enfermedad

\footnotetext{
Escuela de medicina, Universidad de Ciencias Aplicadas. Lima, Perú. Centro Nacional de Epidemiologia Prevención y Control de Enfermedades. Ministerio de Salud. Lima, Perú.

Estudiante de medicina; ${ }^{\mathrm{b}}$ médico epidemiólogo

Recibido: 15/11/2017 Aprobado: 22/12/2017 En línea: 15/05/2018
}

Citar como: Chávez-Ramos M, Munayco CV, Soto-Cabezas G. Características de la enfermedad tuberculosa en estudiantes de la salud de Perú. Rev Peru Med Exp Salud Publica. 2018;35(2): 350-1.doi: 10.17843/rpmesp.2018.352.3247.
Tabla 1. Características de enfermedad tuberculosa en estudiantes de ciencias de la salud de Perú 2013-2016

\begin{tabular}{|c|c|c|}
\hline Características & $\mathbf{n}$ & $\%$ \\
\hline \multicolumn{3}{|l|}{ Años de notificación } \\
\hline 2013 & 27 & 25,0 \\
\hline 2014 & 27 & 25,0 \\
\hline 2015 & 33 & 30,6 \\
\hline 2016 & 21 & 19,4 \\
\hline \multicolumn{3}{|l|}{ Sexo } \\
\hline Hombre & 34 & 31,5 \\
\hline Mujer & 74 & 68,5 \\
\hline Edad promedio & $23 \pm 5$ & \\
\hline \multicolumn{3}{|l|}{ Carrera universitaria/técnica } \\
\hline Enfermería & 23 & 21,1 \\
\hline Medicina & 27 & 24,8 \\
\hline Obstetricia & 11 & 10,1 \\
\hline Odontología & 8 & 7,3 \\
\hline $\begin{array}{l}\text { Otras carreras (biología, farmacia, } \\
\text { asistencia social y tecnología médica) }\end{array}$ & 13 & 11,9 \\
\hline Técnica/o de enfermería & 27 & 24,8 \\
\hline \multicolumn{3}{|l|}{ Localización de la tuberculosis } \\
\hline Tuberculosis Pulmonar & 81 & 75,0 \\
\hline Negativo & 24 & 29,6 \\
\hline Positivo(+) & 34 & 42,0 \\
\hline Positivo(++) & 12 & 14,8 \\
\hline Positivo(+++) & 11 & 13,6 \\
\hline Tuberculosis extrapulmonar & 27 & 25,0 \\
\hline Negativo & 22 & 81,5 \\
\hline No se realizó & 3 & 11,1 \\
\hline Resultado pendiente & 2 & 7,4 \\
\hline \multicolumn{3}{|l|}{ Tuberculosis resistente } \\
\hline Tuberculosis MDR & 6 & 75,0 \\
\hline Tuberculosis XDR & 1 & 12,5 \\
\hline Tuberculosis Monoresistente & 1 & 12,5 \\
\hline
\end{tabular}

tuberculosa extra pulmonar, de los cuales siete estudiantes presentaron tuberculosis multidrogrorresistente (TB MDR).

Se concluye que los estudiantes de salud son una población de alto riesgo de enfermar por tuberculosis y dentro de este grupo los más afectados son los estudiantes de medicina, debido probablemente a que presentan actividades académicas en establecimientos de salud y por ello están en constante exposición con la enfermedad, a pesar de que no trabajan de manera fija en el centro de salud ${ }^{(5)}$. Por lo que se debe fortalecer las medidas de control de infecciones para reducir el riesgo de enfermar por tuberculosis.

Contribuciones de los autores: MCR participó en la concepción y diseño del artículo, análisis e interpretación de datos; redacción del artículo; revisión crítica del artículo; aprobación de la versión final. CVM participó en el análisis e interpretación de datos; redacción del artículo, aprobación de la versión final. GSC: recolección de datos, revisión crítica del artículo; aprobación de la versión final.

Fuentes de financiamiento: autofinanciada.

Conflicto de Interés: Los autores declaran no tener conflictos de interés. 


\section{REFERENCIAS BIBLIOGRÁFICAS}

1. Organización Panamericana de la Salud. La tuberculosis en la Región de las Américas, Informe Regional. Washington, DC. 2014.

2. Baussano I, Nunn P, Williams B, Pivetta E, Bugiani M, Scano F. Tuberculosis among health care workers. Emerg Infect Dis. 2011;17(3):488-494. doi: 10.3201/eid1703.100947

3. Willingham FF, Schmitz TL, Contreras M, Kalangi SE, Vivar AM, Caviedes L, et al. Hospital control and multidrug-resistant pulmonary tuberculosis in female patients, Lima, Peru. Emerg Infect Dis. 2001;7(1):123-7

4. Bonifacio N, Saito M, Gilman RH, Leung F, Cordova N, Chacaltana J, et al. High Risk for Tuberculosis in Hospital Physicians, Peru. Emerg Infect Dis. 2002;8(7):747-748. doi: $10.3201 /$ eid0807.010506

5. Soto-Cabezas MG, Chávez-Pachas AM, Arrasco-Alegre JC, Yagui-Moscoso MJA. Tuberculosis en trabajadores de salud en el Perú, 2013-2015. Rev Peru Med Exp Salud Publica. 2016;33(4):607-15. doi: 10.17843/rpmesp.2016.334.2542

Correspondencia: Marcelo Chávez Ramos

Dirección: Jr. Ayar Manco 281, Zarate. Lima, Perú

Correo electrónico:vchavezramos002@hotmail.com

\section{DESIGUALDADES SOCIOGEOGRÁFICAS EN LA MORTALIDAD MATERNA EN PERÚ: 2001-2015}

\section{SOCIO-GEOGRAPHIC INEQUALITIES IN MATERNAL MORTALITY IN PERU: 2001-2015}

\author{
Eduardo Casalino Rojo 1,a, Edurne Ochoa Amenabar 1,a , \\ Oscar J. Mújica 2,b , César V. Munayco 1,3,c
}

Sr. Editor. Las desigualdades en salud son causadas por la distribución no equitativa de la riqueza, el poder, los recursos y otros determinantes distales, afectando desproporcionadamente a la población socialmente más vulnerable como, por ejemplo, las madres (1). A nivel mundial, la mortalidad materna es la segunda causa de muerte de mujeres en edad reproductiva, luego del $\mathrm{VIH}{ }^{(2)}$.

\footnotetext{
1 Escuela de Medicina, Universidad Peruana de Ciencias Aplicadas. Lima, Perú.

2 Programa Especial sobre Desarrollo Sostenible y Equidad en Salud, Organización Panamericana de la Salud/Organización Mundial de la Salud. 3 Centro Nacional de Epidemiología Prevención y Control de Enfermedades, Ministerio de Salud. Lima, Perú.

a Estudiante de Medicina; ${ }^{b}$ Médico, magíster en salud pública; ${ }^{\mathrm{c}}$ Médico, magíster en Epidemiología y salud pública, Doctor en Salud Pública Recibido: 15/11/2017 Aprobado: 22/12/2017 En línea: 15/05/2018
}

Citar como: Casalino Rojo E, Ochoa Amenabar E, Mujica OJ, Munayco CV. Desigualdades sociogeográficas en la mortalidad materna en Perú 2001-2015. Rev Peru Med Exp Salud Publica. 2018;35(2): 351-3.doi: 10.17843/ rpmesp.2018.352.3246.
Con el objetivo de explorar si la desigualdad en la razón de mortalidad materna (RMM) se redujo en el Perú durante la ventana temporal de los Objetivos de Desarrollo del Milenio (ODM), se realizó un estudio ecológico de la distribución de la RMM entre departamentos, ordenados según su nivel de ingreso, para el periodo 2001-2015. El número de muertes maternas, el ingreso neto mensual en soles y la población de nacidos vivos para cada departamento y año fueron obtenidos de la serie de Indicadores Básicos del Centro Nacional de Epidemiologia, Prevención y Control de Enfermedades del Ministerio de Salud. A fin de mejorar la robustez de los estimados, se calculó la razón de mortalidad materna promedio en tres periodos trienales para cada departamento: 2001-03, 2007-09 y 2012-15.

La desigualdad sociogeográfica en la RMM en los tres periodos analizados se caracterizó mediante el uso de cuatro métricas estándar: las brechas absoluta y relativa, el índice de desigualdad de la pendiente (IDP) y el índice de concentración en salud (ICS). La brecha absoluta corresponde a la diferencia entre la RMM del cuartil 1 y el cuartil 4 de la distribución departamental de ingreso; la brecha relativa corresponde al cociente entre las RMM de dichos cuartiles extremos. EI IDP, un indicador de gradiente absoluto de desigualdad, corresponde a la pendiente de la regresión lineal por mínimos cuadrados ponderados entre la RMM y la posición social relativa definida por el nivel de ingreso. EI ICS, un indicador de gradiente relativo de desigualdad, se obtiene calculando el área bajo la curva de concentración, que ilustra la desproporcionalidad entre la carga de mortalidad materna y la población de nacidos vivos acumulada según ingreso ${ }^{(3)}$. Los cálculos se realizaron con el Explorador de Equidad de Organización Panamericana de Salud (OPS) ${ }^{(4)}$.

La RMM promedio nacional mostró una modesta reducción en el periodo analizado: de 88,5 por 100000 en 2001-03 bajó a 68,7 por 100000 en 2012-15 (i.e., 22,4\% de reducción). La desigualdad en la RMM según ingreso entre departamentos, mostró una reducción más marcada y sostenida en el mismo periodo: entre 2001-03 y 2012-15, la brecha absoluta se redujo de 103,8 a 52,9 muertes maternas en exceso por 100000 nacidos vivos y la brecha relativa se redujo de 3,9 a 2,3. El IDP se redujo de 147,0 a 72,8 muertes maternas en exceso por 100000 nacidos vivos (i.e., $50.4 \%$ de reducción) y el ICS se redujo de $-0,26$ a de $-0,17$.

La Figura 1 ilustra los resultados de nuestra exploración analítica: se aprecia la horizontalización de la gradiente de RMM entre los cuartiles departamentales de ingreso (Figura 1A) durante el periodo analizado, al igual que la horizontalización de la pendiente de las líneas de regresión (Figura 1B), así como la diagonalización (i.e., acercamiento hacia la línea de equidistribución) de la curva de concentración de la desigualdad en salud (Figura 1C).

Nuestro estudio exploratorio documenta dos tendencias relevantes: por un lado, la regresividad de la desigualdad en la mortalidad materna; es decir, la sistemática concentración de 